\title{
IEDITORIAL
}

\section{Cardio-oncology: the questions to be solved}

\author{
Ana Pardo Sanz, José Luis Zamorano
}

University Hospital Ramón y Cajal, Madrid, Spain

\section{RELATED ARTICLE}

by Tajstra et al, see p. 139

\section{Correspondence to:}

Prof. José Luis Zamorano, University Hospital Ramón y Cajal, Carretera Colmenar Viejo Km 9.100, Madrid, Spain, phone: +34 913360000, email: zamorano@secardiologia.es Received: January 16, 2021. Accepted: January 17, 2021. Published online: February 25, 2021. Kardiol Pol. 2021; 79 (2): 112-113 doi:10.33963/KP.15826 Copyright by the Author(s), 2021
The first thing to consider when creating a service for patients is to define its objectives and functions. The field of cardio-oncology has developed into an important pillar for patients with cancer and (or at risk of) cardiovascular disease. It has experienced exponential growth in the last few years, and there is an important heterogeneity of its functions.

The article "Two professions against two killer diseases: the rationale, organization, and initial experience of a cardio-oncology service" by Tajstra et $\mathrm{al}^{1}$ from this issue of Kardiologia Polska (Kardiol Pol, Polish Heart Journal) describes the initial experience of a cardio-oncology service, in order to serve as a valuable tool for those who are going to develop this field. Their service is designed to include 2 major domains: general cardio-oncology and electrotherapy consultations. All cancer patients referred to the cardio-oncology service between March 2016 and December 2019 were included in the observational study, with information about baseline demographics, cancer type, the reason for referral, cardiac evaluation, and initial clinical outcomes. In $18 \%$ of patients, the reason of referral was cardiovascular complications related to cancer treatment. The most common cancer was breast cancer.

The role of cardiac management in patients undergoing chemotherapy with potential cardiotoxicity is crucial and able to improve the outcomes of these patients. In a recent article by Santoro et $a{ }^{,}{ }^{2}$ female patients with HER2-positive breast cancer undergoing chemotherapy were enrolled. Patients underwent Doppler echocardiography at baseline and every 3 months during cancer therapy. In all patients who developed a drop in left ventricular ejection fraction or global longitudinal strain, cardioprotective treatment was initiated (ramipril and carvedilol). This resulted in left ventricular ejection fraction recovery in most of these patients, and they could finish their cancer therapy.

In the study by Tajstra et al, ${ }^{1}$ there was also a large group of patients with a cardiac implantable electronic device qualified for radiotherapy, with an indication for magnetic resonance imaging (MRI), and with a clinical indication for cancer surgery who were evaluated by the cardio-oncology service.

As a result of aging, the number of patients with cardiac implantable electronic devices requiring radiotherapy is rising. The device can sustain damage due to radiotherapy. ${ }^{3}$

It has been generally agreed that MRI is contraindicated in this group of patients with cardiac implantable electronic devices, including pacemakers, cardiac defibrillators, and loop recorders. ${ }^{4}$ A magnetic wave field is generated during the imaging which can negatively affect the electric components of an electronic device as well as the image produced by the MRI. ${ }^{5}$ The magnetic field generates currents that can displace components within the device and damage it, and it can even potentially generate life-threatening arrhythmias. At least half of the patients with cardiac implantable electronic devices are predicted to have a clinical indication for MRI during their lifetime after the device implantation. ${ }^{6}$

In order to solve this problem, in recent years, the manufacturers of cardiac implantable electronic devices have developed MRI-conditional cardiac implantable electronic devices, which are considered to have minimal risks associated with MRI scans within a specific magnetic resonance environment; however, despite this, clinicians are still hesitant to perform MRIs on patients with cardiac implantable electronic devices because it has historically been considered as a contraindication for MRI. In addition, there is little guidance on how to appropriately perform MRI scans in patients with cardiac implantable electronic devices. The risk of adverse events is 
relatively low and seems to be consistent with the conclusions of the reported studies, including devices that are considered nonconditional.

The European Society of Cardiology Position Paper $^{7}$ summarizes all the available evidence on cardio-oncology with the aim of assisting health professionals in selecting the best management strategies for oncologic patients, considering the impact on outcome, as well as the risk-benefit ratio of particular diagnostic or therapeutic means. It should help health professionals to make decisions in their daily practice.

We should identify the needs and priorities of this group of patients, and observational studies are necessary for this purpose. ${ }^{8}$

A competent cardio-oncology service should include an oncologist, a cardiologist qualified in the field of oncology (possible interactions, anticoagulation and antiplatelet treatment, complications of the treatment, etc.), a cardiologist qualified in cardiac imaging, and a cardiologist qualified in arrhythmias and electrophysiology for patients with pacemakers and other devices. The oncologic patient with or at high risk of cardiovascular disease should be referred to the cardio-oncology service, and protocols should be created and followed so that the process is as systematic as possible and the patients receive the optimal care.

\section{ARTICLE INFORMATION}

DISCLAIMER The opinions expressed by the author(s) are not necessarily those of the journal editors, Polish Cardiac Society, or publisher.

CONFLICT OF INTEREST None declared.

OPEN ACCESS This is an Open Access article distributed under the terms of the Creative Commons Attribution-NonCommercial-NoDerivatives 4.0 International License (CC BY-NC-ND 4.0), allowing third parties to download articles and share them with others, provided the original work is properly cited, not changed in any way, distributed under the same license, and used for noncommercial purposes only. For commercial use, please contact the journal office at kardiologiapolska@ptkardio.pl.

HOW TO CITE Pardo Sanz A, Zamorano JL. Cardio-oncology: the questions to be solved. Kardiol Pol. 2021; 79: 112-113. doi:10.33963/KP.15826

\section{REFERENCES}

1 Tajstra M, Blamek S, Skoczylas I, et al. Two professions against two killer diseases: the rationale, organization, and initial experience of a cardio-oncology service. Kardiol Pol. 2021; 79: 139-146.

2 Santoro C, Esposito R, Lembo M, et al. Strain-oriented strategy for quiding cardioprotection initiation of breast cancer patients experiencing cardiac dysfunction. Eur Hear J - Cardiovasc Imaging. 2019; 39: 1-8.

3 Riva G, Alessandro 0, Spoto R, et al. Radiotherapy in patients with cardiac implantable electronic devices: clinical and dosimetric aspects. Med Oncol. 2018; 35: 73.

4 Korutz AW, Obajuluwa A, Lester MS, et al. Pacemakers in MRI for the Neuroradiologist. AJNR Am J Neuroradiol. 2017; 38: 2222-2230.

5 Verma A, Ha ACT, Dennie C, et al. Canadian Heart Rhythm Society and Canadian Association of Radiologists consensus statement on magnetic resonance imaging with cardiac implantable electronic devices. Can J Cardiol. 2014; 30: 1131-1141. 6 Indik JH, Gimbel JR, Abe H, et al. 2017 HRS expert consensus statement on magnetic resonance imaging and radiation exposure in patients with cardiovascular implantable electronic devices. Hear Rhythm. 2017; 14: e97-e153.

7 Zamorano JL, Lancellotti P, Rodriguez Muñoz D, et al. 2016 ESC Position Paper on cancer treatments and cardiovascular toxicity developed under the auspices of the ESC Committee for Practice Guidelines. Eur Heart J. 2016; 37: 2768-2801.

8 Pardo Sanz A, Rincón L, Guedes Ramallo P, et al. Current status of anticoagulation in patients with breast cancer and atrial fibrillation. Breast. 2019; 46: 163-169. 